\title{
Cohen 弱核多线性算子
}

献给吴从炘教授 85 华诞

黎永锦 1 ，刘琪 ${ }^{1}$, 卜庆营 ${ }^{2 *}$

1. 中山大学数学学院, 广州 510275 ;

2. Department of Mathematics, University of Mississippi, University, MS 38677, USA

E-mail: stslyj@mail.sysu.edu.cn, liuq325@mail2.sysu.edu.cn, qbu@olemiss.edu

收稿日期: 2020-06-08; 接受日期: 2020-08-08; 网络出版日期: 2020-11-17; * 通信作者

国家自然科学基金 (批准号: 11971493) 资助项目

摘要 本文介绍并研究 Banach 空间上一类新的多线性算子, 称为 Cohen 弱 $p$ - 核多线性算子. 本文为 这一类新的多线性算子建立了相应的 Pietsch 控制定理. 作为应用, 本文证明了每个 Cohen 弱 $p$ - 核多 线性算子都是强 $p$ - 可和的并且也是 Cohen 强 $p$ - 可和的.

关键词多线性算子 核算子 $p$ - 可和算子

MSC (2010) 主题分类 $46 \mathrm{G} 25,47 \mathrm{H} 60$

\section{1 引言}

可和线性算子在 Banach 空间理论中起着重要的作用 (参见文献 [1]). 在最近二十年中, 线性算子 的可和性以许多不同的方式扩展到多线性算子的可和性. 例如, 将一类绝对 $p$ - 可和线性算子扩展到 多线性算子的绝对 $p$ - 可和、 $p$ - 控制和强 $p$ - 可和 (参见文献 [2-4]), 以及将一类 Cohen 强 $p$ - 可和和 Cohen $p$ - 核线性算子分别扩展到 Cohen 强 $p$ - 可和和 Cohen $p$ - 核多线性算子 (参见文献 $[5,6]$ ). 本文 将一类 Cohen $p$ - 核线性算子扩展到一类新的多线性算子, 即 Cohen 弱 $p$ - 多线性算子, 并证明这种新 的多线性算子是算子理想, 介于 Cohen 强 $p$ - 可和多线性算子与 Cohen $p$ - 核多线性算子之间. 利用 Ky Fan 引理为 Cohen 弱 $p$ - 核多线性算子建立了 Pietsch 控制定理, 从而得出每个 Cohen 弱 $p$ - 核多线性 算子都是强 $p$ - 可和的. 每个多线性算子 $T: X_{1} \times \cdots \times X_{m} \rightarrow Y$ 在乘积空间上具有投影张量积的线性 化 $\widetilde{T}=X_{1} \hat{\otimes}_{\pi} \cdots \hat{\otimes}_{\pi} X_{m} \rightarrow Y$. 在本文的最后, 分别针对多线性算子的 $p$ - 控制、强 $p$ - 可和、Cohen 强 $p$ - 可和、Cohen $p$ - 核和 Cohen 弱 $p$ - 核, 总结了 $T$ 的可和性与其线性化 $\widetilde{T}$ 的可和性之间的关系. 


\section{2 预备知识}

对于 Banach 空间 $X$, 令 $X^{*}$ 表示其拓扑对偶, 而 $B_{X}$ 表示其闭单位球. 对于 Banach 空间 $X$ 和 $Y$ 以及连续线性算子 $u: X \rightarrow Y$, 令 $u^{*}: Y^{*} \rightarrow X^{*}$ 表示 $u$ 的伴随算子. 对于 $1 \leqslant p<\infty$, 令 $q$ 表示其共轭, 即 $1 / p+1 / q=1$. 令 $\left(e_{n}\right)_{n}$ 表示 $\ell_{p}$ 的单位向量基, 即对于每一个 $n \in \mathbb{N}, e_{n}=(0, \ldots, 0, \stackrel{(n)}{1}, 0,0, \ldots)$. 给定 一个 Banach 空间 $X$ 和 $1 \leqslant p<\infty, X$ 中的序列 $\left(x_{n}\right)_{n}$ 称为 (i) 绝对 $p$ - 可和的, 如果 $\sum_{n=1}^{\infty}\left\|x_{n}\right\|^{p}<\infty$; (ii) 弱 $p$ - 可和的, 如果 $\sum_{n=1}^{\infty}\left|x^{*}\left(x_{n}\right)\right|^{p}<\infty$ 对于每一个 $x^{*} \in X^{*}$ 成立; (iii) 强 $p$ - 可和的, 如果 $\sum_{n=1}^{\infty}\left|x_{n}^{*}\left(x_{n}\right)\right|<\infty$ 对于 $X^{*}$ 中的每一个弱 $q-$ 可和序列 $\left(x_{n}^{*}\right)_{n}$ 成立. 如果 $\left(x_{n}\right)_{n}$ 是 $X$ 中的一个弱 $p-$ 可和序列, 那么 $\left\|\left(x_{n}\right)_{n}\right\|_{\ell_{p}^{w}(X)}:=\sup \left\{\left(\sum_{n=1}^{\infty}\left|x^{*}\left(x_{n}\right)\right|^{p}\right)^{1 / p}: x^{*} \in B_{X^{*}}\right\}<\infty$.

给定 Banach 空间 $X$ 和 $Y$, 线性算子 $u: X \rightarrow Y$ 被称作 (i) 绝对 $p$ - 可和的 $(1 \leqslant p<\infty)$, 如 果 $u$ 将 $X$ 中的弱 $p$ - 可和序列作用为 $Y$ 中的绝对 $p$ - 可和序列, 即存在正常数 $K$ 对于任意有限序 列 $x_{1}, \ldots, x_{n} \in X$ 使得 $\left(\sum_{i=1}^{n}\left\|u x_{i}\right\|^{p}\right)^{1 / p} \leqslant K\left\|\left(x_{i}\right)_{1}^{n}\right\|_{\ell_{p}^{w}(X)}$, 令 $\Pi_{p}(X ; Y)$ 表示从 $X$ 到 $Y$ 上所有绝对 $p$ - 可和算子组成的空间; (ii) Cohen 强 $p$ - 可和的 $(1<p<\infty$ ) (参见文献 [7]), 如果 $u$ 将 $X$ 中的绝 对 $p$ - 可和序列作用为 $Y$ 中的强 $p$ - 可和序列, 即存在正常数 $K$ 对于任意有限序列 $x_{1}, \ldots, x_{n} \in X$ 和 $y_{1}^{*}, \ldots, y_{n}^{*} \in Y^{*}$ 使得 $\sum_{i=1}^{n}\left|\left\langle u x_{i}, y_{i}^{*}\right\rangle\right| \leqslant K\left(\sum_{i=1}^{n}\left\|x_{i}\right\|^{p}\right)^{1 / p}\left\|\left(y_{i}^{*}\right)_{i=1}^{n}\right\|_{\ell_{q}\left(Y^{*}\right)}$, 令 $\mathcal{D}_{p}(X ; Y)$ 表示从 $X$ 到 $Y$ 上所有 Cohen 强 $p$ - 可和算子组成的空间; (iii) Cohen $p$ - 核的 $(1<p<\infty$ ) (参见文献 [7]), 如果 $u$ 将 $X$ 中的弱 $p$ - 可和序列作用为 $Y$ 中的强 $p$ - 可和序列, 即存在正常数 $K$ 对于任意有限序列 $x_{1}, \ldots, x_{n} \in X$ 和 $y_{1}^{*}, \ldots, y_{n}^{*} \in Y^{*}$ 使得 $\sum_{i=1}^{n}\left|\left\langle u x_{i}, y_{i}^{*}\right\rangle\right| \leqslant K\left\|\left(x_{i}\right)_{1}^{n}\right\|_{\ell_{p}^{w}(X)}\left\|\left(y_{i}^{*}\right)_{i=1}^{n}\right\|_{\ell_{q}\left(Y^{*}\right)}$, 令 $\mathcal{N}_{p}(X ; Y)$ 表示从 $X$ 到 $Y$ 上所有 Cohen $p$ - 核算子组成的空间. 我们知道 $u \in \mathcal{D}_{p}(X ; Y)$ 当且仅当 $u^{*} \in \Pi_{q}\left(Y^{*} ; X^{*}\right), u \in \mathcal{N}_{p}(X ; Y)$ 当且仅当 $u^{*} \in \mathcal{N}_{q}\left(Y^{*} ; X^{*}\right)$. 另外, $u \in \mathcal{N}_{p}(X ; Y)$ 当且仅当存在 Banach 空间 $Z, v \in \Pi_{p}(X ; Z)$ 和 $w \in \mathcal{D}_{p}(Z ; Y)$ 使得 $u=w \circ v$ (参见文献 [1]).

对于 Banach 空间 $X_{1}, \ldots, X_{m}$ 和 $Y$, 令 $\mathcal{L}^{m}\left(X_{1}, \ldots, X_{m} ; Y\right)$ 定义为从 $X_{1} \times \cdots \times X_{m}$ 到 $Y$ 上的所 有连续 $m$ - 线性算子组成的空间, 其范数定义为 $\|T\|=\sup \left\{\left\|T\left(x_{1}, \ldots, x_{m}\right)\right\|:\left\|x_{1}\right\| \leqslant 1, \ldots,\left\|x_{m}\right\| \leqslant 1\right\}$. 令 $X_{1} \hat{\otimes}_{\pi} \cdots \hat{\otimes}_{\pi} X_{m}$ 定义为 $X_{1}, \ldots, X_{m}$ 的投影张量积, 那么对于 $T \in \mathcal{L}^{m}\left(X_{1}, \ldots, X_{m} ; Y\right)$ 有唯一的 线性化 $\widetilde{T} \in \mathcal{L}\left(X_{1} \hat{\otimes}_{\pi} \cdots \hat{\otimes}_{\pi} X_{m} ; Y\right)$ 使得 $T=\widetilde{T} \circ \otimes$, 其中 $\otimes: X_{1} \times \cdots \times X_{m} \rightarrow X_{1} \hat{\otimes}_{\pi} \cdots \hat{\otimes}_{\pi} X_{m}$ 由 $\otimes\left(x^{1}, \ldots, x^{m}\right)=x^{1} \otimes \cdots \otimes x^{m}$ 给出, 这里 $\left(x^{1}, \ldots, x^{m}\right) \in X_{1} \times \cdots \times X_{m}$. 另外, $\mathcal{L}^{m}\left(X_{1}, \ldots, X_{m} ; Y\right)$ 是 与 $\mathcal{L}\left(X_{1} \hat{\otimes}_{\pi} \cdots \hat{\otimes}_{\pi} X_{m} ; Y\right)$ 等距同构的. 特别地,

$$
\mathcal{L}^{m}\left(X_{1}, \ldots, X_{m}\right):=\mathcal{L}^{m}\left(X_{1}, \ldots, X_{m} ; \mathbb{R}\right)=\left(X_{1} \hat{\otimes}_{\pi} \cdots \hat{\otimes}_{\pi} X_{m}\right)^{*} .
$$

$m$ - 线性算子 $T: X_{1} \times \cdots \times X_{m} \rightarrow Y$ 被称作:

(i) 绝对 $p$ - 可和的 $(1 \leqslant p<\infty)$ (参见文献 [2]), 如果存在一个正常数 $K$ 使得对于任意 $x_{1}^{j}, \ldots, x_{n}^{j}$ $\in X_{j}(j=1, \ldots, m)$, 有

$$
\left(\sum_{i=1}^{n}\left\|T\left(x_{i}^{1}, \ldots, x_{i}^{m}\right)\right\|^{p}\right)^{1 / p} \leqslant K \prod_{j=1}^{m}\left\|\left(x_{i}^{j}\right)_{i=1}^{n}\right\|_{\ell_{p}^{w}\left(X_{j}\right)} .
$$

令 $\mathcal{L}_{a s, p}^{m}\left(X_{1}, \ldots, X_{m} ; Y\right)$ 定义为从 $X_{1} \times \cdots \times X_{m}$ 到 $Y$ 上所有绝对 $p$ - 可和 $m$ - 线性算子组成的空间. 显然, $\mathcal{L}_{a s, p}^{1}(X ; Y)=\Pi_{p}(X ; Y)$.

(ii) $p$ - 控制的 $(1 \leqslant p<\infty)$ (参见文献 [3]), 如果存在一个正常数 $K$ 使得对于任意 $x_{1}^{j}, \ldots, x_{n}^{j} \in X_{j}$ $(j=1, \ldots, m)$, 有

$$
\left(\sum_{i=1}^{n}\left\|T\left(x_{i}^{1}, \ldots, x_{i}^{m}\right)\right\|^{p / m}\right)^{m / p} \leqslant K \prod_{j=1}^{m}\left\|\left(x_{i}^{j}\right)_{i=1}^{n}\right\|_{\ell_{p}^{w}\left(X_{j}\right)} .
$$


令 $\mathcal{L}_{d, p}^{m}\left(X_{1}, \ldots, X_{m} ; Y\right)$ 定义为从 $X_{1} \times \cdots \times X_{m}$ 到 $Y$ 上所有 $p$ - 控制 $m$ - 线性算子组成的空间. 显然, $\mathcal{L}_{d, p}^{1}(X ; Y)=\Pi_{p}(X ; Y)$.

(iii) 强 $p$ - 可和的 $(1 \leqslant p<\infty)$ (参见文献 [4]), 如果存在一个正常数 $K$ 使得对于任意 $x_{1}^{j}, \ldots, x_{n}^{j}$ $\in X_{j}(j=1, \ldots, m)$, 有

$$
\left(\sum_{i=1}^{n}\left\|T\left(x_{i}^{1}, \ldots, x_{i}^{m}\right)\right\|^{p}\right)^{1 / p} \leqslant K \sup _{\phi \in B_{\mathcal{L}^{m}\left(X_{1}, \ldots, X_{m}\right)}}\left(\sum_{i=1}^{n}\left|\phi\left(x_{i}^{1}, \ldots, x_{i}^{m}\right)\right|^{p}\right)^{1 / p} .
$$

令 $\mathcal{L}_{s s, p}^{m}\left(X_{1}, \ldots, X_{m} ; Y\right)$ 定义为从 $X_{1} \times \cdots \times X_{m}$ 到 $Y$ 上所有强 $p$ - 可和 $m$ - 线性算子组成的空间. 显 然, $\mathcal{L}_{s s, p}^{1}(X ; Y)=\Pi_{p}(X ; Y)$.

(iv) Cohen 强 $p$ - 可和的 $(1<p<\infty)$ (参见文献 [5]), 如果存在一个正常数 $K$ 使得对于任意 $x_{1}^{j}, \ldots, x_{n}^{j} \in X_{j}(j=1, \ldots, m)$ 和 $y_{1}^{*}, \ldots, y_{n}^{*} \in Y^{*}$, 有

$$
\sum_{i=1}^{n}\left|\left\langle T\left(x_{i}^{1}, \ldots, x_{i}^{m}\right), y_{i}^{*}\right\rangle\right| \leqslant K\left(\sum_{i=1}^{n} \prod_{j=1}^{m}\left\|x_{i}^{j}\right\|^{p}\right)^{1 / p}\left\|\left(y_{i}^{*}\right)_{i=1}^{n}\right\|_{\ell_{q}^{w}\left(Y^{*}\right)} .
$$

令 $\mathcal{D}_{p}^{m}\left(X_{1}, \ldots, X_{m} ; Y\right)$ 定义为从 $X_{1} \times \cdots \times X_{m}$ 到 $Y$ 上所有 Cohen 强 $p$ - 可和 $m$ - 线性算子组成的空 间. 显然, $\mathcal{D}_{p}^{1}(X ; Y)=\mathcal{D}_{p}(X ; Y)$.

(v) Cohen $p$ - 核的 $(1<p<\infty)$ (参见文献 [6]), 如果存在一个正常数 $K$ 使得对于任意 $x_{1}^{j}, \ldots, x_{n}^{j}$ $\in X_{j}(j=1, \ldots, m)$ 和 $y_{1}^{*}, \ldots, y_{n}^{*} \in Y^{*}$, 有

$$
\sum_{i=1}^{n}\left|\left\langle T\left(x_{i}^{1}, \ldots, x_{i}^{m}\right), y_{i}^{*}\right\rangle\right| \leqslant K\left(\sup _{x^{j *} \in B_{X_{j}^{*}}} \sum_{i=1}^{n} \prod_{j=1}^{m}\left|\left\langle x_{i}^{j}, x^{j *}\right\rangle\right|^{p}\right)^{1 / p}\left\|\left(y_{i}^{*}\right)_{i=1}^{n}\right\|_{\ell_{q}^{w}\left(Y^{*}\right)} .
$$

令 $\mathcal{N}_{p}^{m}\left(X_{1}, \ldots, X_{m} ; Y\right)$ 定义为从 $X_{1} \times \cdots \times X_{m}$ 到 $Y$ 上所有 Cohen $p$ - 核 $m$ - 线性算子组成的空间. 显然, $\mathcal{N}_{p}^{1}(X ; Y)=\mathcal{N}_{p}(X ; Y)$.

\section{Cohen 弱 $p$ - 核算子}

定义 $3.1 m$ - 线性算子 $T: X_{1} \times \cdots \times X_{m} \rightarrow Y$ 被称作 Cohen 弱 $p$ - 核的 $(1<p<\infty)$, 如果存 在正常数 $K$ 使得对于任意 $x_{1}^{j}, \ldots, x_{n}^{j} \in X_{j}(j=1, \ldots, m)$ 和 $y_{1}^{*}, \ldots, y_{n}^{*} \in Y^{*}$, 有下式成立:

$$
\sum_{i=1}^{n}\left|\left\langle T\left(x_{i}^{1}, \ldots, x_{i}^{m}\right), y_{i}^{*}\right\rangle\right| \leqslant K \cdot \sup _{\phi \in B_{\mathcal{L}^{m}\left(X_{1}, \ldots, X_{m}\right)}}\left(\sum_{i=1}^{n}\left|\phi\left(x_{i}^{1}, \ldots, x_{i}^{m}\right)\right|^{p}\right)^{1 / p} \cdot\left\|\left(y_{i}^{*}\right)_{i=1}^{n}\right\|_{\ell_{q}^{w}\left(Y^{*}\right)} .
$$

令 $\mathcal{N}_{w, p}^{m}\left(X_{1}, \ldots, X_{m} ; Y\right)$ 定义为从 $X_{1} \times \cdots \times X_{m}$ 到 $Y$ 上所有 Cohen 弱 $p$ - 核 $m$ - 线性算子组成的 空间, 并且在范数 $n_{w, p}^{m}(T)$ 下是 Banach 空间, 其中 $n_{w, p}^{m}(T)$ 是使得 $(3.1)$ 成立的最小常数 $K$. 显然, $\mathcal{N}_{w, p}^{1}(X ; Y)=\mathcal{N}_{p}(X ; Y)$.

对于 Cohen 弱 $p$ - 核多线性算子, 可以得到以下性质.

命题 3.1 如果 $T \in \mathcal{N}_{w, p}^{m}\left(X_{1}, \ldots, X_{m} ; Y\right), u_{j} \in \mathcal{L}\left(E_{j} ; X_{j}\right)$, 其中 $j=1, \ldots, m, v \in L(Y ; Z)$, 那么 $v \circ T \circ\left(u_{1}, \ldots, u_{m}\right) \in \mathcal{N}_{w, p}^{m}\left(E_{1}, \ldots, E_{m}, Z\right)$.

注意到 $\mathcal{L}^{m}\left(X_{1}, \ldots, X_{m}\right)=\left(X_{1} \hat{\otimes}_{\pi} \cdots \hat{\otimes}_{\pi} X_{m}\right)^{*}$. 如果 $\left(x_{i}^{1} \otimes \cdots \otimes x_{i}^{m}\right)_{i}$ 是 $X_{1} \hat{\otimes}_{\pi} \cdots \hat{\otimes}_{\pi} X_{m}$ 中的一 个弱 $p$ - 可和序列, 那么对于每一个 $\phi \in \mathcal{L}^{m}\left(X_{1}, \ldots, X_{m}\right)$, 有 $\sum_{i=1}^{\infty}\left|\phi\left(x_{i}^{1}, \ldots, x_{i}^{m}\right)\right|^{p}=\sum_{i=1}^{\infty} \mid \widetilde{\phi}\left(x_{i}^{1} \otimes \cdots\right.$ $\left.\otimes x_{i}^{m}\right)\left.\right|^{p}<\infty$. 从定义可知 $T: X_{1} \times \cdots \times X_{m} \rightarrow Y$ 是 Cohen 弱 $p$ - 核的当且仅当 $\left(x_{i}^{1} \otimes \cdots \otimes x_{i}^{m}\right)_{i}$ 是 
$X_{1} \hat{\otimes}_{\pi} \cdots \hat{\otimes}_{\pi} X_{m}$ 中的一个弱 $p$ - 可和序列时 $\left(\widetilde{T}\left(x_{i}^{1} \otimes \cdots \otimes x_{i}^{m}\right)\right)_{i}$ 是 $Y$ 中的一个强 $p$ - 可和序列. 然而, 我们不知道如果 $T$ 是 Cohen 弱 $p$ - 核的能否得到 $\widetilde{T}$ 是 Cohen $p$ - 核的.

注意到对于任意 $\phi \in \mathcal{L}^{m}\left(X_{1}, \ldots, X_{m}\right),\left|\phi\left(x^{1}, \ldots, x^{m}\right)\right| \leqslant\|\phi\|\left\|x^{1}\right\| \cdots\left\|x^{m}\right\|$. 对于 $x^{j *} \in B_{X_{j}^{*}}(1 \leqslant j$ $\leqslant m)$, 令 $\phi_{1}=x^{1 *} \otimes \cdots \otimes x^{m *}$. 那么 $\phi_{1} \in B_{\mathcal{L}^{m}\left(X_{1}, \ldots, X_{m}\right)}$ 和 $\phi_{1}\left(x^{1}, \ldots, x^{m}\right)=\prod_{j=1}^{m}\left\langle x^{j}, x^{j *}\right\rangle$. 因此, 以 下结果可以直接从定义得到.

命题 3.2 令 $1<p<\infty$, 则

$$
\mathcal{N}_{p}^{m}\left(X_{1}, \ldots, X_{m} ; Y\right) \subseteq \mathcal{N}_{w, p}^{m}\left(X_{1}, \ldots, X_{m} ; Y\right) \subseteq \mathcal{D}_{p}^{m}\left(X_{1}, \ldots, X_{m} ; Y\right)
$$

每个 $T \in \mathcal{N}_{p}^{m}\left(X_{1}, \ldots, X_{m} ; Y\right)$ 是弱紧算子 (参见文献 [6]), 并且 $T \in \mathcal{D}_{p}^{m}\left(X_{1}, \ldots, X_{m} ; Y\right)$ 是弱紧的 (参见文献 [8]). 因此 $T \in \mathcal{N}_{w, p}^{m}\left(X_{1}, \ldots, X_{m} ; Y\right)$ 也是弱紧的.

定理 $3.1 m$ - 线性算子 $T: X_{1} \times \cdots \times X_{m} \rightarrow Y$ 是 Cohen 弱 $p$ - 核的当且仅当存在正常数 $K$ 及 $\left(B_{\mathcal{L}^{m}\left(X_{1}, \ldots, X_{m}\right)}, w^{*}\right)$ 上的正则概率测度 $\mu$ 和 $\left(B_{Y^{* *}}, w^{*}\right)$ 上的 $\lambda$ 使得对于任意 $x^{j} \in X_{j}(1 \leqslant j \leqslant m)$ 和 $y^{*} \in Y^{*}$, 有

$$
\begin{aligned}
\left|\left\langle T\left(x^{1}, \ldots, x^{m}\right), y^{*}\right\rangle\right| \leqslant & K\left(\int_{B_{\mathcal{L}^{m}\left(X_{1}, \ldots, X_{m}\right)}}\left|\phi\left(x^{1}, \ldots, x^{m}\right)\right|^{p} d \mu(\phi)\right)^{1 / p} \\
& \times\left(\int_{B_{Y^{* *}}}\left|y^{* *}\left(y^{*}\right)\right|^{q} d \lambda\left(y^{* *}\right)\right)^{1 / q} .
\end{aligned}
$$

证明 在这里令 $\mathcal{L}^{m}:=\mathcal{L}^{m}\left(X_{1}, \ldots, X_{m}\right)$. 为了证明充分性, 假设 (3.2) 成立, 对于 $1 \leqslant i \leqslant n$ 和 $1 \leqslant j \leqslant m$, 取任意的 $x_{i}^{j} \in X_{j}, y_{i}^{*} \in Y^{*}$. 根据 (3.2) 和 Hölder 不等式, 有

$$
\begin{aligned}
\sum_{i=1}^{n}\left|\left\langle T\left(x_{i}^{1}, \ldots, x_{i}^{m}\right), y_{i}^{*}\right\rangle\right| \leqslant & K\left(\int_{B_{\mathcal{L}^{m}}} \sum_{i=1}^{n}\left|\phi\left(x_{i}^{1}, \ldots, x_{i}^{m}\right)\right|^{p} d \mu(\phi)\right)^{1 / p} \\
& \times\left(\int_{B_{Y^{* *}}} \sum_{i=1}^{n}\left|y^{* *}\left(y_{i}^{*}\right)\right|^{q} d \lambda\left(y^{* *}\right)\right)^{1 / q} \\
\leqslant & K \sup _{\phi \in B_{\mathcal{L}^{m}}}\left(\sum_{i=1}^{n}\left|\phi\left(x_{i}^{1}, \ldots, x_{i}^{m}\right)\right|^{p}\right)^{1 / p}\left\|\left(y_{i}^{*}\right)_{i=1}^{n}\right\|_{\ell_{q}^{w}\left(Y^{*}\right)},
\end{aligned}
$$

因此, $T$ 是 Cohen 弱 $p$ - 核的.

为了证明必要性, 根据文献 [5,6] 中的方法并且结合 Ky Fan 引理 (参见文献 [1]). 假设 $T: X_{1} \times$ $\cdots \times X_{m} \rightarrow Y$ 是 Cohen 弱 $p$ - 核的. 令 $\Omega_{1}=\left(B_{\mathcal{L}^{m}}, w^{*}\right)$ 和 $\Omega_{2}=\left(B_{Y^{* *}}, w^{*}\right)$, 则 $E:=\left(C\left(\Omega_{1}\right)^{*}, w^{*}\right)$ $\times\left(C\left(\Omega_{2}\right)^{*}, w^{*}\right)$ 是一个 Hausdorff 拓扑向量空间且 $\mathcal{C}:=B_{C\left(\Omega_{1}\right)^{*}} \times B_{C\left(\Omega_{2}\right)^{*}}$ 是 $E$ 中的紧凸子集. 令 $M$ 为 $\mathcal{C}$ 上的所有实值函数且具有下面形式:

$$
\begin{aligned}
f(\mu, \lambda)= & \sum_{i=1}^{n}\left|\left\langle T\left(x_{i}^{1}, \ldots, x_{i}^{m}\right), y_{i}^{*}\right\rangle\right|-\frac{K}{p} \sum_{i=1}^{n} \int_{B_{\mathcal{L}^{m}}}\left|\phi\left(x_{i}^{1}, \ldots, x_{i}^{m}\right)\right|^{p} d \mu(\phi) \\
& -\frac{K}{q} \sum_{i=1}^{n} \int_{B_{Y^{* *}}}\left|y^{* *}\left(y_{i}^{*}\right)\right|^{q} d \lambda\left(y^{* *}\right)
\end{aligned}
$$

对于 $1 \leqslant i \leqslant n$ 和 $1 \leqslant j \leqslant m$, 其中 $x_{i}^{j} \in X_{j}, y_{i}^{*} \in Y^{*}$. 很明显, 这样的函数是凸的且连续的. 对于任意 的 $0 \leqslant \alpha \leqslant 1$ 和具有 (3.3) 形式的函数 $f$ 和 $g$, 容易得到 $\alpha f+(1-\alpha) g$ 也具有 (3.3) 这样的形式 (参 
见文献 [5]). 因而, $M$ 是一个凸集. 注意到 $C\left(\Omega_{1}\right)$ 和 $C\left(\Omega_{2}\right)$ 中的每一个元素是范数可达的. 对于具有 (3.3) 形式的函数, 存在 $\phi_{0} \in B_{\mathcal{L}^{m}}$ 和 $y_{0}^{* *} \in B_{Y^{* *}}$ 使得

$$
\left(\sum_{i=1}^{n}\left|\phi_{0}\left(x_{i}^{1}, \ldots, x_{i}^{m}\right)\right|^{p}\right)^{1 / p}=\sup _{\phi \in B_{\mathcal{L}^{m}}}\left(\sum_{i=1}^{n}\left|\phi\left(x_{i}^{1}, \ldots, x_{i}^{m}\right)\right|^{p}\right)^{1 / p}
$$

和

$$
\left(\sum_{i=1}^{n}\left|y_{0}^{* *}\left(y_{i}^{*}\right)\right|^{q}\right)^{1 / q}=\sup _{y^{* *} \in B_{Y^{* *}}}\left(\sum_{i=1}^{n}\left|y^{* *}\left(y_{i}^{*}\right)\right|^{q}\right)^{1 / q}=\left\|\left(y_{i}^{*}\right)_{i=1}^{n}\right\|_{\ell_{q}^{w}\left(Y^{*}\right)} .
$$

在下面基本等式中:

$$
a b=\inf _{\epsilon>0}\left\{\frac{1}{p}\left(\frac{a}{\epsilon}\right)^{p}+\frac{1}{q}(\epsilon b)^{q}\right\}, \quad \forall a, b>0
$$

令

$$
a=\left(\sum_{i=1}^{n}\left|\phi_{0}\left(x_{i}^{1}, \ldots, x_{i}^{m}\right)\right|^{p}\right)^{1 / p}, \quad b=\left(\sum_{i=1}^{n}\left|y_{0}^{* *}\left(y_{i}^{*}\right)\right|^{q}\right)^{1 / q} .
$$

令 $\delta_{y_{0}^{* *}}$ 和 $\delta_{\phi_{0}}$ 表示 Dirac 测度, 那么应用 $(3.4)$, 其中 $\epsilon=1$, 有

$$
\begin{aligned}
f\left(\delta_{\phi_{0}}, \delta_{y_{0}^{* *}}\right) & =\sum_{i=1}^{n}\left|\left\langle T\left(x_{i}^{1}, \ldots, x_{i}^{m}\right), y_{i}^{*}\right\rangle\right|-\frac{K}{p} \sum_{i=1}^{n}\left|\phi_{0}\left(x_{i}^{1}, \ldots, x_{i}^{m}\right)\right|^{p}-\frac{K}{q} \sum_{i=1}^{n}\left|y_{0}^{* *}\left(y_{i}^{*}\right)\right|^{q} \\
& \leqslant \sum_{i=1}^{n}\left|\left\langle T\left(x_{i}^{1}, \ldots, x_{i}^{m}\right), y_{i}^{*}\right\rangle\right|-K a b \\
& =\sum_{i=1}^{n}\left|\left\langle T\left(x_{i}^{1}, \ldots, x_{i}^{m}\right), y_{i}^{*}\right\rangle\right|-K \sup _{\phi \in B_{\mathcal{L}^{m}}}\left(\sum_{i=1}^{n}\left|\phi\left(x_{i}^{1}, \ldots, x_{i}^{m}\right)\right|^{p}\right)^{1 / p}\left\|\left(y_{i}^{*}\right)_{i=1}^{n}\right\|_{\ell_{q}^{w}\left(Y^{*}\right)} \\
& \leqslant 0 .
\end{aligned}
$$

根据 Ky Fan 引理, 对于任意 $f \in M$, 存在 $(\mu, \lambda) \in \mathcal{C}$ 使得 $f(\mu, \lambda) \leqslant 0$. 如果 $f$ 是由单元素 $\left(x^{1}, \ldots, x^{m}\right) \in$ $X_{1} \times \cdots \times X_{m}$ 生成, 另外有单元素 $y^{*} \in Y^{*}$, 那么根据 $(3.3)$ 有

$$
\left|\left\langle T\left(x^{1}, \ldots, x^{m}\right), y^{*}\right\rangle\right| \leqslant \frac{K}{p} \int_{B_{\mathcal{L}^{m}}}\left|\phi\left(x^{1}, \ldots, x^{m}\right)\right|^{p} d \mu(\phi)+\frac{K}{q} \int_{B_{Y^{* *}}}\left|y^{* *}\left(y^{*}\right)\right|^{q} d \lambda\left(y^{* *}\right) .
$$

再次使用基本等式并令

$$
a=\left(\int_{B_{\mathcal{L}^{m}}}\left|\phi\left(x^{1}, \ldots, x^{m}\right)\right|^{p} d \mu(\phi)\right)^{1 / p}, \quad b=\left(\int_{B_{Y^{* *}}}\left|y^{* *}\left(y^{*}\right)\right|^{q} d \lambda\left(y^{* *}\right)\right)^{1 / q},
$$

那么对于任意的 $\epsilon>0$, 有

$$
\begin{aligned}
\left|\left\langle T\left(x^{1}, \ldots, x^{m}\right), y^{*}\right\rangle\right|= & \left|\left\langle T\left(\epsilon^{-1 / m} x^{1}, \ldots, \epsilon^{-1 / m} x^{m}\right), \epsilon y^{*}\right\rangle\right| \\
\leqslant & \frac{K}{p} \int_{B_{\mathcal{L}^{m}}}\left|\phi\left(\epsilon^{-1 / m} x^{1}, \ldots, \epsilon^{-1 / m} x^{m}\right)\right|^{p} d \mu(\phi) \\
& +\frac{K}{q} \int_{B_{Y^{* *}}}\left|y^{* *}\left(\epsilon y^{*}\right)\right|^{q} d \lambda\left(y^{* *}\right) \\
= & \frac{K}{p}\left(\frac{a}{\epsilon}\right)^{p}+\frac{K}{q}(\epsilon b)^{q} .
\end{aligned}
$$


在 (3.4) 中对所有的 $\epsilon>0$ 取下确界, 那么有

$$
\left|\left\langle T\left(x^{1}, \ldots, x^{m}\right), y^{*}\right\rangle\right| \leqslant K\left(\int_{B_{\mathcal{L}^{m}}}\left|\phi\left(x^{1}, \ldots, x^{m}\right)\right|^{p} d \mu(\phi)\right)^{1 / p}\left(\int_{B_{Y^{* *}}}\left|y^{* *}\left(y^{*}\right)\right|^{q} d \lambda\left(y^{* *}\right)\right)^{1 / q},
$$

因此 (3.2) 成立. 证明完毕.

对于强可和多线性算子, 将定理 3.1 与 Pietsch 控制定理相结合 (参见文献 [4]), 有以下结果.

命题 3.3 令 $1<p<\infty$, 则

$$
\mathcal{N}_{w, p}^{m}\left(X_{1}, \ldots, X_{m} ; Y\right) \subseteq \mathcal{L}_{s s, p}^{m}\left(X_{1}, \ldots, X_{m} ; Y\right) .
$$

Achour 和 Alouani ${ }^{[6]}$ 给出了 Cohen $p$ - 核多线性算子的因式分解. 现在, 将因式分解重新写为以 下形式.

命题 3.4 设 $T: X_{1} \times \cdots \times X_{m} \rightarrow Y$ 是 $m$ - 线性算子且 $1<p<\infty$, 则 $T$ 是 Cohen $p$ - 核的当且 仅当存在 Banach 空间 $Z 、 p$ - 控制 $m$ - 线性算子 $S: X_{1} \times \cdots \times X_{m} \rightarrow Z$ 和 Cohen 强 $p$ - 可和线性算子 $u: Z \rightarrow Y$ 使得 $T=u \circ S$, 即 $\mathcal{N}_{p}^{m}=D_{p} \circ \mathcal{L}_{d, p}^{m}$.

证明 充分性. 选取任意的 $x^{1} \in X_{1}, \ldots, x^{m} \in X_{m}$ 和任意的 $y^{*} \in Y^{*}$. 根据 Cohen 强 $p$ - 可和算 子和 $p$ - 控制算子的 Pietsch 控制定理 (参见文献 [5]), 有

$$
\begin{aligned}
\mid\left\langle T\left(x^{1}, \ldots, x^{m}\right), y^{*}\right\rangle & =\left\langle u\left(S\left(x^{1}, \ldots, x^{m}\right)\right), y^{*}\right\rangle \\
& \leqslant K_{1}\left\|S\left(x^{1}, \ldots, x^{m}\right)\right\|_{Z} \cdot\left\|y^{*}\right\|_{L_{q}\left(B_{\left.Y^{* *}, \lambda\right)}\right.} \\
& \leqslant K_{1} K_{2} \prod_{j=1}^{m}\left\|x^{j}\right\|_{L_{p}\left(B_{X_{j}^{*}}, \mu_{j}\right)} \cdot\left\|y^{*}\right\|_{L_{q}\left(B_{\left.Y^{* *}, \lambda\right)},\right.}
\end{aligned}
$$

那么根据文献 [6, 定理 2.5] 可以得到 $T$ 是 Cohen $p$ - 核的.

必要性. 根据文献 $\left[6\right.$, 定理 3.1], 存在 Banach 空间 $G_{1}, \ldots, G_{m}$ 、绝对 $p$ - 可和线性算子 $u_{j}: X_{j} \rightarrow G_{j}$ $(1 \leqslant j \leqslant m)$ 和 Cohen 强 $p$ - 可和 $m$ - 线性算子 $R: G_{1} \times \cdots \times G_{m} \rightarrow Y$ 使得 $T=R \circ\left(u_{1}, \ldots, u_{m}\right)$. 令 $S=\otimes \circ\left(u_{1}, \ldots, u_{m}\right)$ 和 $u=\widetilde{R}$, 那么有下面的关系:

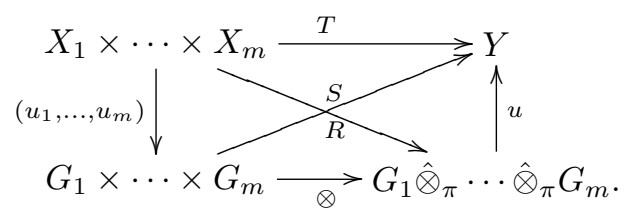

于是可得 $u$ 是 Cohen 强 $p$ - 可和的 (参见文献 $[8,9]$ ) 并且 $S$ 是 $p$ - 控制的 (参见文献 $[5,8]$ ). 综上, 证明 完毕.

下面的问题是有趣的, 即是否可以通过强 $p$ - 可和多线性算子和 Cohen 强 $p$ - 可和线性算子来分解 每个 Cohen 弱 $p$ - 核多线性算子, 这是一个未解决的问题. 但是, 对于相反的情形, 我们有以下结果.

定理 3.2 设 $T: X_{1} \times \cdots \times X_{m} \rightarrow Y$ 是 $m$ - 线性算子且 $1<p<\infty$.

(i) $T$ 是 Cohen $p$ - 核的;

(ii) $T$ 是 Cohen 弱 $p$ - 核的;

(iii) $\widetilde{T}: X_{1} \hat{\otimes}_{\pi} \cdots \hat{\otimes}_{\pi} X_{m} \rightarrow Y$ 是 Cohen $p$ - 核的;

(iv) 存在 Banach 空间 $Z$ 、强 $p$ - 可和 $m$ - 线性算子 $S: X_{1} \times \cdots \times X_{m} \rightarrow Z$ 和 Cohen 强 $p$ - 可和线 性算子 $u: Z \rightarrow Y$ 使得 $T=u \circ S$.

那么 (i) $\Rightarrow$ (iv) $\nRightarrow$ (i) 并且 (iii) $\Rightarrow$ (iv) $\Rightarrow$ (ii). 
证明 (i) $\Rightarrow$ (iv) 因为 $p$ - 控制 $m$ - 线性算子是强 $p$ - 可和的, (iv) 可由命题 3.4 得到.

(iv) $\nRightarrow$ (i) 参阅下面例 3.1 中的情形 1 .

(iii) $\Rightarrow$ (iv) 因为 $\widetilde{T}$ 是 Cohen $p$-核的, 存在 Banach 空间 $Z$ 、绝对 $p$ - 可和线性算子 $v: X_{1} \hat{\otimes}_{\pi} \cdots \hat{\otimes}_{\pi} X_{m}$ $\rightarrow Z$ 和 Cohen 强 $p$ - 可和线性算子 $u: Z \rightarrow Y$ 使得 $\widetilde{T}=u \circ v$. 令 $S=v \circ \otimes$, 则 $T=\widetilde{T} \circ \otimes=u \circ v \circ \otimes=u \circ S$ 并有下面的关系:

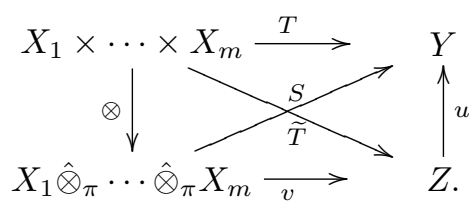

因为 $\widetilde{S}=v$, 所以可得 $S$ 是强 $p$ - 可和的 (参见文献 [4]). 因此, 可得 (iv) 成立.

(iv) $\Rightarrow$ (ii) 选取任意的 $x^{1} \in X_{1}, \ldots, x^{m} \in X_{m}$ 和任意的 $y^{*} \in Y^{*}$. 对于 Cohen 强 $p$ - 可和算子和 强 $p$ - 可和算子, 根据 Pietsch 控制定理, 有

$$
\begin{aligned}
\left|\left\langle T\left(x^{1}, \ldots, x^{m}\right), y^{*}\right\rangle\right| & =\left\langle u\left(S\left(x^{1}, \ldots, x^{m}\right)\right), y^{*}\right\rangle \\
& \leqslant K_{1}\left\|S\left(x^{1}, \ldots, x^{m}\right)\right\|_{Z} \cdot\left\|y^{*}\right\|_{L_{q}\left(B_{\left.Y^{* *}, \lambda\right)}\right.} \\
& \leqslant K_{1} K_{2}\left\|\left(x^{1}, \ldots, x^{m}\right)\right\|_{L_{p}\left(B_{\mathcal{L}^{m}\left(X_{1}, \ldots, X_{m}\right)}, \mu\right)} \cdot\left\|y^{*}\right\|_{L_{q}\left(B_{Y^{* *}}, \lambda\right)},
\end{aligned}
$$

根据定理 3.1 可得 $T$ 是 Cohen 弱 $p$ - 可和的. 综上, 证明完毕.

公开问题 在定理 3.2 条件下, 是否有 $(\mathrm{i}) \Rightarrow$ (iii) 成立?

Achour 和 Alouani ${ }^{[6]}$ 构造了多线性算子, 它是 Cohen $p$ - 核的, 因此也是 Cohen 弱 $p$ - 核的. 在下 面的例 3.1 情形 1 中, 将构造多线性算子, 其为 Cohen 弱 $p$ - 核而不是 Cohen $p$ - 核的. 接下来证明对 于特殊的 Banach 空间, Cohen $p$ - 核多线性算子与 Cohen 弱 $p$ - 核多线性算子的一致性.

命题 3.5 如果 $X_{1}$ 和 $X_{2}$ 是 $\mathcal{L}_{\infty^{-}}$空间, $Y$ 是任意的 Banach 空间, 那么对于任意的 $p \geqslant 2$, 有 $\mathcal{N}_{p}^{2}\left(X_{1}, X_{2} ; Y\right)=\mathcal{N}_{w, p}^{2}\left(X_{1}, X_{2} ; Y\right)$.

证明 假设 $X_{1}$ 和 $X_{2}$ 分别是 $\mathcal{L}_{\infty, \sigma_{1}}$ - 空间和 $\mathcal{L}_{\infty, \sigma_{2}}$ 空间. 根据文献 [10, 推论 4.4] 可知, 每一个 双线性形式 $\phi: X_{1} \times X_{2} \rightarrow \mathbb{R}$ 是 2- 控制的, 因此, 对于 $p \geqslant 2$ 也是 $p$ - 控制的, 且有 $\|\phi\|_{d, p} \leqslant\|\phi\|_{d, 2}$ $\leqslant \sigma_{1} \sigma_{2} K_{G}\|\phi\|$, 其中 $K_{G}$ 是 Grothendieck 常数. 选取任意的 $T \in \mathcal{N}_{w, p}^{2}\left(X_{1}, X_{2} ; Y\right), x^{1} \in X_{1}, x^{2} \in X_{2}$, $y^{*} \in Y^{*}$. 对于控制多线性算子, 根据 Pietsch 控制定理 (参见文献 [5]), 有

$$
\begin{aligned}
\left|\phi\left(x^{1}, x^{2}\right)\right| & \leqslant\|\phi\|_{d, p} \cdot\left\|x^{1}\right\|_{L_{p}\left(B_{\left.X_{1}^{*}, \mu_{1}\right)}\right.} \cdot\left\|x^{2}\right\|_{L_{p}\left(B_{\left.X_{2}^{*}, \mu_{2}\right)}\right.} \\
& \leqslant \sigma_{1} \sigma_{2} K_{G}\|\phi\| \cdot\left\|x^{1}\right\|_{L_{p}\left(B_{\left.X_{1}^{*}, \mu_{1}\right)}\right)} \cdot\left\|x^{2}\right\|_{L_{p}\left(B_{\left.X_{2}^{*}, \mu_{2}\right)}\right.} .
\end{aligned}
$$

根据定理 3.1 有

$$
\begin{aligned}
\left|\left\langle T\left(x^{1}, x^{2}\right), y^{*}\right\rangle\right| & \leqslant K\left(\int_{B_{\mathcal{L}^{2}\left(X_{1}, X_{2}\right)}}\left|\phi\left(x^{1}, x^{2}\right)\right|^{p} d \mu(\phi)\right)^{1 / p} \cdot\left\|y^{*}\right\|_{L_{q}\left(B_{\left.Y^{* *}, \lambda\right)}\right.} \\
& \leqslant \sigma_{1} \sigma_{2} K K_{G}\left\|x^{1}\right\|_{L_{p}\left(B_{\left.X_{1}^{*}, \mu_{1}\right)}\right)} \cdot\left\|x^{2}\right\|_{L_{p}\left(B_{X_{2}^{*}}, \mu_{2}\right)} \cdot\left\|y^{*}\right\|_{L_{q}\left(B_{Y^{* *}}, \lambda\right)},
\end{aligned}
$$

那么对于 Cohen 核多线性算子, 根据 Pietsch 控制定理 (参见文献 [6]), 可得 $T \in \mathcal{N}_{p}^{2}\left(X_{1}, X_{2} ; Y\right)$.

例 3.1 定义 $T_{1}: \ell_{2} \times \ell_{2} \rightarrow \ell_{1}$ 为 $T_{1}(x, y)=\left(x_{k} y_{k}\right)_{k}$, 其中 $x=\left(x_{k}\right)_{k}, y=\left(y_{k}\right)_{k} \in \ell_{2}$, 那么 $T_{1} \in \mathcal{L}^{2}\left(\ell_{2}, \ell_{2} ; \ell_{1}\right)$. 令 $I_{1}: \ell_{1} \hookrightarrow \ell_{2}$ 是典态包含映射且 $T_{2}=I_{1} \circ T_{1}: \ell_{2} \times \ell_{2} \rightarrow \ell_{2}$; 对于任意的 $p \geqslant 1, T_{2}$ 
是强 $p$ - 可和的 (参见文献 [10]). 令 $I_{2}: \ell_{2} \hookrightarrow \ell_{\infty}$ 是典态包含映射且 $T_{3}=I_{2} \circ T_{2}: \ell_{2} \times \ell_{2} \rightarrow \ell_{\infty}$. 因为 对于任意 $p \geqslant 1, I_{1}$ 是绝对 $p$ - 可和的, $I_{2}=I_{1}^{*}$; 对于任意的 $p>1, I_{2}$ 是 Cohen 强 $p$ - 可和的. 因此, $T_{3}$ 满足定理 3.2(iv), 所以对于任意 $p>1, T_{3}$ 是 Cohen 弱 $p$ - 核的.

情形 1 对于任意的 $p>1, T_{3}$ 不是 Cohen $p$ - 核的.

假设 $T_{3} \in \mathcal{N}_{p}^{2}\left(\ell_{2}, \ell_{2} ; \ell_{\infty}\right)$, 则对于任意 $n \in \mathbb{N}$, 存在正常数 $K$, 使得

$$
\sum_{i=1}^{n}\left|\left\langle T_{3}\left(e_{i}, e_{i}\right), e_{i}\right\rangle\right| \leqslant K\left(\sup _{\phi^{1}, \phi^{2} \in B_{\ell_{2}}} \sum_{i=1}^{n} \prod_{j=1}^{2}\left|\left\langle e_{i}, \phi^{j}\right\rangle\right|^{p}\right)^{1 / p} \cdot\left\|\left(e_{i}\right)_{i=1}^{n}\right\|_{\ell_{q}^{w}\left(\ell_{\infty}\right)} .
$$

注意到 (参见文献 [11]) 如果 $\left(x_{i}^{*}\right)_{i}$ 是 $X^{*}$ 中弱 $q$ - 可和序列, 则

$$
\left\|\left(x_{i}^{*}\right)_{i}\right\|_{\ell_{q}^{w}\left(X^{*}\right)}=\sup \left\{\left(\sum_{i=1}^{\infty}\left|x_{i}^{*}(x)\right|^{q}\right)^{1 / q}: x \in B_{X}\right\} .
$$

因此,

$$
\left\|\left(e_{i}\right)_{i=1}^{n}\right\|_{\ell_{q}^{w}\left(\ell_{\infty}\right)}=\sup _{\phi \in B_{\ell_{1}}}\left(\sum_{i=1}^{n}\left|\left\langle e_{i}, \phi\right\rangle\right|^{q}\right)^{1 / q} \leqslant \sup _{\phi \in B_{\ell_{1}}}\|\phi\|_{\ell_{q}} \leqslant \sup _{\phi \in B_{\ell_{1}}}\|\phi\|_{\ell_{1}} \leqslant 1 .
$$

于是对于任意的 $\phi^{1}, \phi^{2} \in B_{\ell_{2}}$, 有

$$
\begin{aligned}
\left(\sum_{i=1}^{n} \prod_{j=1}^{2}\left|\left\langle e_{i}, \phi^{j}\right\rangle\right|^{p}\right)^{1 / p} & =\left(\sum_{i=1}^{n}\left|\phi_{i}^{1} \phi_{i}^{2}\right|^{p}\right)^{1 / p} \leqslant \sum_{i=1}^{n}\left|\phi_{i}^{1} \phi_{i}^{2}\right| \\
& \leqslant\left(\sum_{i=1}^{n}\left|\phi_{i}^{1}\right|^{2}\right)^{1 / 2}\left(\sum_{i=1}^{n}\left|\phi_{i}^{2}\right|^{2}\right)^{1 / 2} \\
& \leqslant\left\|\phi^{1}\right\|_{\ell_{2}} \cdot\left\|\phi^{2}\right\|_{\ell_{2}} \leqslant 1 .
\end{aligned}
$$

但是 $\sum_{i=1}^{n}\left|\left\langle T_{3}\left(e_{i}, e_{i}\right), e_{i}\right\rangle\right|=n$. 所以导出矛盾, 即得 $T_{3} \notin \mathcal{N}_{p}^{2}\left(\ell_{2}, \ell_{2} ; \ell_{\infty}\right)$.

情形 2 对于任意的 $p \geqslant 2, T_{2}$ 不是 $p$ - 控制的.

假设 $T_{2} \in \mathcal{L}_{d, p}^{2}\left(\ell_{2}, \ell_{2} ; \ell_{2}\right)$, 则对于任意 $n \in \mathbb{N}$, 存在正常数 $K$, 使得

$$
\left(\sum_{i=1}^{n}\left\|T\left(e_{i}, e_{i}\right)\right\|^{p / 2}\right)^{2 / p} \leqslant K\left\|\left(e_{i}\right)_{i=1}^{n}\right\|_{\ell_{p}^{w}\left(\ell_{2}\right)}^{2} .
$$

注意到

$$
\left\|\left(e_{i}\right)_{i=1}^{n}\right\|_{\ell_{p}^{w}\left(\ell_{2}\right)}^{2}=\sup _{\phi \in B_{\ell_{2}}}\left(\sum_{i=1}^{n}\left|\left\langle e_{i}, \phi\right\rangle\right|^{p}\right)^{2 / p} \leqslant \sup _{\phi \in B_{\ell_{2}}} \sum_{i=1}^{n}\left|\left\langle e_{i}, \phi\right\rangle\right|^{2} \leqslant 1,
$$

但是 $\left(\sum_{i=1}^{n}\left\|T\left(e_{i}, e_{i}\right)\right\|^{p / 2}\right)^{2 / p}=n^{2 / p}$, 所以导出矛盾, 即得 $T_{2} \notin \mathcal{L}_{d, p}^{2}\left(\ell_{2}, \ell_{2} ; \ell_{2}\right)$.

例 3.2 定义 $T_{4}: \ell_{1} \times \ell_{1} \rightarrow \ell_{1}$ 为 $T_{1}(x, y)=\left(x_{k} y_{k}\right)_{k}$, 其中 $x=\left(x_{k}\right)_{k}, y=\left(y_{k}\right)_{k} \in \ell_{1}$, 则对于任意 $p \geqslant 1, T_{4}$ 是强 $p$ - 可和的, 但是 $T_{4}$ 不是弱紧的 (参见文献 [10]).

情形 3 对于任意 $p \geqslant 2, T_{4}$ 是 $p$ - 控制的.

选取 $x^{i}=\left(x_{k}^{i}\right)_{k}, y^{i}=\left(y_{k}^{i}\right)_{k} \in \ell_{1}$, 其中 $i=1, \ldots, n$. 根据 Khinchin 不等式 (参见文献 [1]), 可得

$$
\left(\sum_{i=1}^{n}\left\|T\left(x^{i}, y^{i}\right)\right\|^{p / 2}\right)^{2 / p}=\left(\sum_{i=1}^{n}\left(\sum_{k=1}^{\infty}\left|x_{k}^{i} y_{k}^{i}\right|\right)^{p / 2}\right)^{2 / p}
$$




$$
\begin{aligned}
& \leqslant\left(\sum_{i=1}^{n}\left(\sum_{k=1}^{\infty}\left|x_{k}^{i}\right|^{2}\right)^{p / 4}\left(\sum_{k=1}^{\infty}\left|y_{k}^{i}\right|^{2}\right)^{p / 4}\right)^{2 / p} \\
& \leqslant \frac{1}{A_{p}^{2}}\left(\sum_{i=1}^{n}\left(\int_{0}^{1}\left|\sum_{k=1}^{\infty} r_{k}(t) x_{k}^{i}\right|^{p} d t\right)^{1 / 2}\left(\int_{0}^{1}\left|\sum_{k=1}^{\infty} r_{k}(t) y_{k}^{i}\right|^{p} d t\right)^{1 / 2}\right)^{2 / p} \\
& \leqslant \frac{1}{A_{p}^{2}}\left(\left(\sum_{i=1}^{n} \int_{0}^{1}\left|\sum_{k=1}^{\infty} r_{k}(t) x_{k}^{i}\right|^{p} d t\right)^{1 / 2}\left(\sum_{i=1}^{n} \int_{0}^{1}\left|\sum_{k=1}^{\infty} r_{k}(t) y_{k}^{i}\right|^{p} d t\right)^{1 / 2}\right)^{2 / p} \\
& =\frac{1}{A_{p}^{2}}\left(\int_{0}^{1} \sum_{i=1}^{n}\left|\left\langle x^{i},\left(r_{k}(t)\right)_{k}\right\rangle\right|^{p} d t\right)^{1 / p}\left(\int_{0}^{1} \sum_{i=1}^{n}\left|\left\langle y^{i},\left(r_{k}(t)\right)_{k}\right\rangle\right|^{p} d t\right)^{1 / p} \\
& \leqslant \frac{1}{A_{p}^{2}}\left\|\left(x^{i}\right)_{i=1}^{n}\right\|_{\ell_{p}^{w}\left(\ell_{1}\right)} \cdot\left\|\left(y^{i}\right)_{i=1}^{n}\right\|_{\ell_{p}^{w}\left(\ell_{1}\right)}
\end{aligned}
$$

其中 $A_{p}$ 是 Khinchin 不等式中的常数, $r_{k}(t)$ 是 Rademacher 函数. 因此, 对于任意 $p \geqslant 2$,

$$
T_{4} \in \mathcal{L}_{d, p}^{2}\left(\ell_{1}, \ell_{1} ; \ell_{1}\right)
$$

令 $T: X_{1} \times \cdots \times X_{m} \rightarrow Y$ 是 $m$ - 线性算子, $\widetilde{T}: X_{1} \hat{\otimes}_{\pi} \cdots \hat{\otimes}_{\pi} X_{m} \rightarrow Y$ 是它的线性化. 关于 $T$ 的可 和性与 $\widetilde{T}$ 的可和性关系, 我们列出下面一些事实.

事实 $3.1 T \in \mathcal{D}_{p}^{m} \Leftrightarrow \widetilde{T} \in \mathcal{D}_{p}^{1}=\mathcal{D}_{p}$ (参见文献 $[8,9]$ ).

事实 $3.2 \widetilde{T} \in \mathcal{L}_{s s, p}^{1}=\Pi_{p} \Rightarrow T \in \mathcal{L}_{s s, p}^{m}$, 但是 $T \in \mathcal{L}_{s s, p}^{m} \nRightarrow \widetilde{T} \in \mathcal{L}_{s s, p}^{1}=\Pi_{p}$ (参见文献 $[4,10]$ ).

事实 $3.3 \widetilde{T} \in \mathcal{L}_{d, p}^{1}=\Pi_{p} \nRightarrow T \in \mathcal{L}_{d, p}^{m}$ 和 $T \in \mathcal{L}_{d, p}^{m} \nRightarrow \widetilde{T} \in \mathcal{L}_{d, p}^{1}=\Pi_{p}$.

根据情形 2 , 可得 $T_{2} \notin \mathcal{L}_{d, p}^{m}$. 但是 $\widetilde{T}_{2}=I_{1} \circ \widetilde{T}_{1} \in \Pi_{p}$. 由情形 $3, T_{4} \in \mathcal{L}_{d, p}^{m}$. 但是 $T_{4}$ 不是弱紧的, 因 此, $\widetilde{T}_{4}$ 不是弱紧的, 所以, $\widetilde{T}_{4} \notin \Pi_{p}$.

事实 $3.4 \widetilde{T} \in \mathcal{N}_{w, p}^{1}=\mathcal{N}_{p} \Rightarrow T \in \mathcal{N}_{w, p}^{m}$ (参见定理 3.2).

事实 $3.5 \widetilde{T} \in \mathcal{N}_{p}^{1}=\mathcal{N}_{p} \nRightarrow T \in \mathcal{N}_{p}^{m}$.

事实上, 根据情形 1 , 可得 $T_{3} \notin \mathcal{N}_{p}^{m}$. 但是因为 $\widetilde{T}_{2} \in \Pi_{p}, I_{2} \in \mathcal{D}_{p}$, 所以 $\widetilde{T}_{3}=I_{2} \circ \widetilde{T}_{2} \in \mathcal{N}_{p}$.

公开问题 $3.1 T \in \mathcal{N}_{w, p}^{m}$ 可否推出 $\widetilde{T} \in \mathcal{N}_{w, p}^{1}=\mathcal{N}_{p}$ ? $T \in \mathcal{N}_{p}^{m}$ 可否推出 $\widetilde{T} \in \mathcal{N}_{p}^{1}=\mathcal{N}_{p}$ ?

致谢 感谢吴从炘教授一直以来的关心和支持, 感谢审稿人的建议.

\section{参考文献}

1 Diestel J, Jarchow H, Tonge A. Absolutely Summing Operators. Cambridge: Cambridge University Press, 1995

2 Matos M C. Absolutely summing holomorphic mappings. An Acad Brasil Ciênc, 1996, 68: 1-13

3 Meléndez Y, Tonge A. Polynomials and the Pietsch domination theorem. Math Proc R Ir Acad, 1999, 2: 195-212

4 Dimant V. Strongly $p$-summing multilinear operators. J Math Anal Appl, 2003, 278: 182-193

5 Achour D, Mezrag L. On the Cohen strongly p-summing multilinear operators. J Math Anal Appl, 2007, 327: 550-563

6 Achour D, Alouani A. On multilinear generalizations of the concept of nuclear operators. Colloq Math, 2010, 120: $85-102$

7 Cohen J S. Absolutely p-summing, p-nuclear operators and their conjugates. Math Ann, 1973, 201: 177-200

8 Mezrag L, Saadi K. Inclusion and coincidence properties for Cohen strongly summing multilinear operators. Collect Math, 2013, 64: 395-408

9 Bu Q, Shi Z. On Cohen almost summing multilinear operators. J Math Anal Appl, 2013, 401: 174-181

10 Carando D, Dimant V. On summability of bilinear operators. Math Nachr, 2003, 259: 3-11

11 Bu Q, Buskes G. Schauder decompositions and the Fremlin projective tensor product of Banach lattices. J Math Anal Appl, 2009, 355: 335-351 


\section{On Cohen weakly nuclear multilinear operators}

Yongjin Li, Qi Liu \& Qingying Bu

Abstract In this paper, we introduce and study a new class of multilinear operators on Banach spaces, called Cohen weakly $p$-nuclear multilinear operators. We establish a Pietsch domination theorem for this new class of multilinear operators. As an application, we show that every Cohen weakly $p$-nuclear multilinear operator is strongly $p$-summing and Cohen strongly $p$-summing.

Keywords multilinear operator, nuclear operator, $p$-summing operator

$\operatorname{MSC}(2010) \quad 46 \mathrm{G} 25,47 \mathrm{H} 60$

doi: $10.1360 /$ SSM-2020-0177 